\title{
The leading effect of fluid inertia on the motion of rigid bodies at low Reynolds number
}

\author{
By A. M. LESHANSKY ${ }^{1}$, O. M. LAVRENTEVA AND A. NIR ${ }^{2}$ \\ ${ }^{1}$ Division of Chemistry \& Chemical Engineering, California Institute of Technology, \\ Pasadena, CA 91125, USA \\ ${ }^{2}$ Department of Chemical Engineering, Technion - IIT, Haifa 32000, Israel
}

(Received 18 January 2002 and in revised form 11 December 2003)

We investigate the influence of fluid inertia on the motion of a finite assemblage of solid spherical particles in slowly changing uniform flow at small Reynolds number, $R e$, and moderate Strouhal number, $S l$. We show that the first effect of fluid inertia on particle velocities for times much larger than the viscous time scales as $\sqrt{S l R e}$ given that the Stokeslet associated with the disturbance flow field changes with time. Our theory predicts that the correction to the particle motion from that predicted by the zero-Re theory has the form of a Basset integral. As a particular example, we calculate the Basset integral for the case of two unequal particles approaching (receding) with a constant velocity along the line of their centres. On the other hand, when the Stokeslet strength is independent of time, the first effect of fluid inertia reduces to a higher order of magnitude and scales as Re. This condition is fulfilled, for example, in the classical problem of sedimentation of particles in a constant gravity field.

\section{Introduction}

The understanding and modelling of the complex flows of dispersed material is paramount in the design and process control of various technological applications, such as fluidization, sedimentation, slurry transportation etc. While the transient or oscillatory motion of a single particle has attracted wide attention through the years (Tchen 1947; Mei \& Adrian 1992; Lovalenti \& Brady 1993; Chang \& Maxey 1994; Coimbra \& Rangel 2001), the theory concerning the analogous behaviour of multiparticle systems is still quite limited, probably due to analytical complexity arising from many-body interactions and the time-dependence of the flow domain geometry. Machu et al. (2001) considered the behaviour of single drops and pairs of drops made from dilute suspensions of microscopic particles with negligible effects of inertia and found a remarkable analogy with the motion of drops of homogeneous liquids. Leichtberg et al. (1976) developed a strong-interaction theory to describe the coaxial gravitational settling of three rigid spheres at low Reynolds numbers, Re. It was suggested that the unsteady Basset history-type contribution to the Stokes drag force in the long-time limit results from the slow temporal change of the domain geometry due to inter-particle interactions. Although they claim that a precise treatment of this force for a multi-particle problem would need to take into account the instantaneous boundaries of the other particles, the classical single-sphere Basset term (Landau \& Lifshitz 1988) was retained in the equation of motion for each particle. Their results show the deviation from the zero-Re theory grows as $t^{1 / 2}$ due to the cumulative effect of the Basset force. Feng \& Joseph (1995) studied the unsteady motion of solid bodies 
in more complex flows. They argued that in cases where the unsteadiness is caused by hydrodynamic inter-particle or wall-particle interactions, the unsteady inertial terms, $\partial \boldsymbol{v} / \partial t$ dominate over the convective ones, $\boldsymbol{v} \cdot \boldsymbol{\nabla} \boldsymbol{v}$. By means of direct numerical simulations of the transient Stokes equations they showed that the unsteady forces may completely change the dynamics of the particles' motion in some cases. Although they mention that if the entire fluid inertia is considered the solution may look quite different from what they have obtained taking into account the unsteady fluid inertia only, the question of when the nonlinear convective inertia terms should be retained in the governing equations remained open.

Hinch \& Nitsche (1993) showed that mutual interactions between fluctuating colloidal particles may give rise to a nonlinear drift force when the fluid inertia is not entirely neglected. Moreover, they found that this force, when integrated over all the spectrum of frequencies, results in a net drift interaction on ensemble average. The nonlinearities due to time-dependence of the flow domain and the convection within the bulk fluid were neglected by considering oscillations with small amplitudes.

Recently, Pieńkowska (2001) studied the structure of an unsteady flow generated in a viscous fluid by a finite number of solid spheres translating in it with prescribed velocities at small Re. A multiple scattering approach was used to describe the effects of mutual non-stationary interactions in terms of velocity tensors and induced forces distributed on the sphere surfaces. The leading-order effect of the unsteady inertia on the forces induced on the particles was found. The fully linearized problem was treated in both this work and Hinch \& Nitsche (1993), i.e. the relative positions of the particles were assumed to be independent of time during the time interval under consideration. Thus, their results are applicable to small time intervals and to the case of small-amplitude particle oscillation.

In the most recent study Coimbra et al. (2004) investigated experimentally history effects in particle response to a high-frequency small-amplitude oscillatory flow. The theoretically determined behaviour of the Basset drag on a single particle freely moving in a unform time-dependent background flow from the earlier studies has been unequivocally validated in these experiments. Their results also show that in the case of the high-frequency background flow the hydrodynamic interaction between particles is negligible even for moderate separation distances of the order of particle size.

This paper presents a preliminary attempt to analyse the leading effect of fluid inertia on the motion of a finite assemblage of rigid spherical particles in a uniform time-dependent flow at low Reynolds numbers during the time interval when the mutual positions of the particles may significantly change. Our focus is on the case of a moderate number of particles in the swarm when the change in the mutual positions may have a substantial effect on the dynamics. The study is performed by means of singular perturbation methods based on the well-established theory of matched asymptotic expansions. It is predicted that as a result of unsteadiness there might be an inertial contribution to the particles' velocities proportional to $\sqrt{S l R e}$, where $\mathrm{Sl}$ is the Strouhal number, given that the net Stokes drag force exerted on them by the fluid varies considerably with time. Some remarks concerning the influence of the fluid inertia on motion of particles suspended in more general time-dependent flows, such as linear and parabolic flows are also included in the discussion.

\section{Statement of the problem}

Consider the evolution of a swarm of $n$ rigid particles of mass $m_{i}$ in a spatially uniform time-dependent unbounded flow of a viscous Newtonian fluid with density 
and the viscosity equal to $\rho$ and $\mu$, respectively. Let $\boldsymbol{x}=\left(x_{1}, x_{2}, x_{3}\right)$ be a radius vector to a point in the laboratory coordinate system with some chosen origin. If we suppose that the particles are spherical, then the domain occupied by the continuous phase may be represented by $\mathscr{D}(t):\left\{\boldsymbol{x} \in \mathfrak{R}^{3}:\left|\boldsymbol{x}-\boldsymbol{Z}_{i}(t)\right|>a_{i}, i=1,2, \ldots, n\right\}$, where $\boldsymbol{Z}_{i}(t)$ denotes a radius vector to the center of the $i$ th particle and $a_{i}$ stands for the particle radius. The size of the swarm can be characterized by $L(t)=\max _{i, j}\left|\boldsymbol{Z}_{i}(t)-\boldsymbol{Z}_{j}(t)\right|$. The velocity field $\boldsymbol{v}(\boldsymbol{x}, t)=\left(v_{1}, v_{2}, v_{3}\right)$ and the pressure $p(\boldsymbol{x}, t)$ are described by the following equations and boundary conditions:

$$
\begin{aligned}
& \partial \boldsymbol{v} / \partial t+\boldsymbol{v} \cdot \nabla \boldsymbol{v}=-\rho^{-1} \nabla p+v \Delta \boldsymbol{v}+\boldsymbol{g}, \quad \boldsymbol{x} \in \mathscr{D}(t), \\
& \nabla \cdot v=0, \quad x \in \mathscr{D}(t), \\
& \boldsymbol{v}=\boldsymbol{u}_{i}(\boldsymbol{x}, t), \quad \boldsymbol{x} \in \Gamma_{i}(t), \\
& \boldsymbol{v} \rightarrow \boldsymbol{v}^{\infty}(t), \quad p \rightarrow p^{\infty}(\boldsymbol{x}, t) \\
& \text { as }|\boldsymbol{x}-\boldsymbol{X}(t)| \rightarrow \infty, \\
& \boldsymbol{v}(\boldsymbol{x}, 0)=\mathbf{0},
\end{aligned}
$$

where $\Gamma_{i}(t)$ denotes the surface of the $i$ th particle, $\boldsymbol{u}_{i}(\boldsymbol{x}, t)$ is the velocity at points on the surface of $i$ th particle, and $\boldsymbol{X}(t)$ is the position vector for the centre of mass of the swarm. We also assume that $\left\{p^{\infty}(\boldsymbol{x}, t), \boldsymbol{v}^{\infty}(t)\right\}$ is a solution of (1)-(2) in $\mathfrak{R}^{3}$ with $\boldsymbol{v}^{\infty}(0)=0$. Since the particles are rigid, $\boldsymbol{u}_{i}=\boldsymbol{U}_{i}(t)+\boldsymbol{\Omega}_{i}(t) \times \boldsymbol{r}_{i}$, where $\boldsymbol{U}_{i}$ and $\boldsymbol{\Omega}_{i}$ are the linear and angular velocities of motion and $\boldsymbol{r}_{i}=\boldsymbol{x}-\boldsymbol{Z}_{i}(t)$ is the radius vector with its origin at the centre of the $i$ th particle. We also assume that the body force is spatially homogeneous and time-dependent with density $\boldsymbol{g}(t)$. The instantaneous force, $\boldsymbol{F}_{i}$, and torque, $\boldsymbol{T}_{i}$, exerted on the $i$ th particle by the flow are given by

$$
\begin{aligned}
\boldsymbol{F}_{i} & =\oint_{\Gamma_{\mathrm{i}}} \boldsymbol{\sigma} \cdot \boldsymbol{n} \mathrm{d} s, \\
\boldsymbol{T}_{i} & =\oint_{\Gamma_{\mathrm{i}}} \boldsymbol{r}_{i} \times(\boldsymbol{\sigma} \cdot \boldsymbol{n}) \mathrm{d} s,
\end{aligned}
$$

where $\sigma=-p \boldsymbol{I}+\mu\left(\nabla \boldsymbol{v}+(\nabla \boldsymbol{v})^{T}\right)$ is the stress tensor. The evolution of the particle swarm is governed by following kinematic conditions:

$$
\boldsymbol{U}_{i}=\dot{\boldsymbol{Z}}_{i}(t), \quad i=1,2, \ldots, n,
$$

where the over-dot stands for the time derivative.

There are two classical problems associated with the equations outlined above:

(i) The resistance problem: to determine forces and torques exerted by the flow on each particle for a given set of particle velocities, $\left\{\boldsymbol{U}_{i}(t), \boldsymbol{\Omega}_{i}(t)\right\}$.

(ii) The mobility problem: to determine $\boldsymbol{U}_{i}(t)$ and $\boldsymbol{\Omega}_{i}(t)$ of particles that are freely suspended in the ambient fluid, for prescribed external forces and torques.

For problem (ii) the dynamic force and torque balances must also be specified,

$$
\begin{aligned}
m_{i} \frac{\mathrm{d} \boldsymbol{U}_{i}}{\mathrm{~d} t} & =\boldsymbol{F}_{i}+m_{i} \boldsymbol{g}, \\
I_{i} \frac{\mathrm{d} \boldsymbol{\Omega}_{\mathrm{i}}}{\mathrm{d} t} & =\boldsymbol{T}_{i},
\end{aligned}
$$

where $I_{i}$ denotes the moment of inertia of particle $i$. To complete the formulation of problem (ii), the initial data for the positions and velocities of the particles should be provided:

$$
\boldsymbol{Z}_{i}(0)=\boldsymbol{Z}_{i 0}, \boldsymbol{U}_{i}(0)=\mathbf{0}, \boldsymbol{\Omega}_{i}(0)=\mathbf{0}, \quad i=1,2, \ldots, n .
$$


In this paper we shall address both problems formulated above with a special attention given to the mobility problem (ii).

There are three different timescales associated with the problem formulated above that are important for our analysis: the viscous relaxation time on the lengthscale associated with the swarm, $t_{v}=L^{2} / v$; the Stokes time associated with the motion of a single particle under the influence of a body force, $t_{S}=v / \operatorname{ag}(\lambda-1)$, where $g=\max |\boldsymbol{g}(t)|$, and $\lambda=\rho_{s} / \rho$ is a typical density ratio; and the time related to the temporal change in the external forces or uniform flow, $t_{e x t}$ (period in the periodic case). Previous theoretical studies of the multi-particle problem (Hinch \& Nitsche 1993; Pieńkowska 2001) were focused on the case when the particles' mutual positions may be regarded as independent of time. The first influence of inertia in this case is described by the fully linearized unsteady Stokes equations. In contrast to this, our focus is on the larger timescales, when the evolution of the geometry of the swarm cannot be neglected.

The following generalized scaling is chosen: the radius of the reference particle, $a$, for length; the characteristic velocity associated with either the particle motion or applied body forces, $\widetilde{u}$, for velocity; $\widetilde{u} / a$ for angular velocity; and $\mu \widetilde{u} / a$ for pressure. The characteristic time $\tau$ is associated either with the slow change (to compare with a viscous time) of the flow due to the motion of particle, $\tau=t_{S}$, or with the variation of the external force, $\tau=t_{e x t} \leqslant O\left(t_{S}\right)$. Assume further that $t_{v} \ll \tau$, i.e. that the quasi-steady velocity field in the vicinity of the swarm is immediately established.

In dimensionless form the undisturbed velocity and pressure fields should satisfy

$$
\operatorname{ReSl} \frac{\partial \boldsymbol{v}^{\infty}}{\partial t}=-\nabla p^{\infty}(\boldsymbol{x}, t)+\eta(t), \quad \boldsymbol{x} \in \mathfrak{R}^{3},
$$

where $R e=\widetilde{u} a / v$ is a Reynolds number, $S l=(a / \widetilde{u}) / \tau$ is a Strouhal number and $\eta=g /\left(v \widetilde{u} / a^{2}\right)$ stands for the dimensionless density of the body force. Note that if the external force and flow do not change with time and the unsteadiness of the problem is caused solely by the evolution of the geometry, then $S l=1$.

Following Lovalenti \& Brady (1993), we pose the problem formulation in a comoving coordinate frame with its origin at the instantaneous centre of a reference particle belonging to the swarm, i.e.

$$
\boldsymbol{x}^{\prime}=\boldsymbol{x}-\int_{0}^{t} \boldsymbol{U}(\tau) \mathrm{d} \tau,
$$

where $\boldsymbol{U}$ denotes the translational velocity of the reference particle. Since we want to focus on a velocity field that vanishes far from the swarm, we consider the disturbance velocity and pressure fields and introduce the new variables

$$
\boldsymbol{v}^{\prime}=\boldsymbol{v}-\boldsymbol{v}^{\infty}, \quad p^{\prime}=p-p^{\infty} .
$$

Thus, after omitting the primes, the problem formulated for the disturbance field in dimensionless form is

$$
\begin{array}{cl}
\operatorname{Re}\left(\operatorname{Sl} \partial \boldsymbol{v} / \partial t+\boldsymbol{v} \cdot \nabla \boldsymbol{v}+\boldsymbol{f}\left(\boldsymbol{v}, \boldsymbol{v}^{\infty}\right)\right)=-\nabla p+\Delta \boldsymbol{v}, & \boldsymbol{x} \in \mathscr{D}(t), \\
\nabla \cdot \boldsymbol{v}=0, & \boldsymbol{x} \in \mathscr{D}(t), \\
\boldsymbol{v}=\boldsymbol{u}_{i}-\boldsymbol{v}^{\infty}, & \boldsymbol{x} \in \Gamma_{i}(t), \\
\boldsymbol{v}, p \rightarrow 0 & \text { as }|\boldsymbol{x}| \rightarrow \infty,
\end{array}
$$

where

$$
f\left(v, v^{\infty}\right)=\left(v^{\infty}-U\right) \cdot \nabla v .
$$


Kinematic conditions (8) and initial conditions (11) in dimensionless form become

$$
\begin{aligned}
& \dot{\boldsymbol{Z}}_{i}(t)=S l^{-1} \boldsymbol{U}_{i}, \quad i=1,2, \ldots, n, \\
& \boldsymbol{Z}_{i}(0)=\boldsymbol{Z}_{i 0}, \boldsymbol{U}_{i}(0)=\mathbf{0}, \boldsymbol{\Omega}_{i}(0)=\mathbf{0}, \quad i=1,2, \ldots, n, \\
& \boldsymbol{v}(\boldsymbol{x}, 0)=\mathbf{0}, \quad \boldsymbol{x} \in \mathscr{D}(0),
\end{aligned}
$$

while the force and torque balances (9)-(10) combined with (6)-(7) become

$$
\begin{gathered}
\widetilde{m}_{i} \operatorname{ReS} l \frac{\mathrm{d} \boldsymbol{U}_{i}}{\mathrm{~d} t}=\oint_{\Gamma_{\mathrm{i}}}\left(\boldsymbol{\sigma}+\boldsymbol{\sigma}^{\infty}\right) \cdot \boldsymbol{n} \mathrm{d} s+\widetilde{m}_{i} \boldsymbol{\eta}(t), \\
\widetilde{I}_{i} \operatorname{Re} S l \frac{\mathrm{d} \boldsymbol{\Omega}_{i}}{\mathrm{~d} t}=\oint_{\Gamma_{\mathrm{i}}} \boldsymbol{r}_{i} \times\left[\left(\boldsymbol{\sigma}+\boldsymbol{\sigma}^{\infty}\right) \cdot \boldsymbol{n}\right] \mathrm{d} s,
\end{gathered}
$$

where $\widetilde{m}_{i}$ is the mass of particle $i$ non-dimensionalized by the product $\rho a^{3}, \widetilde{I}_{i}$ is the moment of inertia of the particle scaled by the product $\rho a^{5}$ and $\sigma^{\infty}$ is the stress tensor corresponding to $\boldsymbol{v}^{\infty}$ and $p^{\infty}$.

\section{Construction of the solution for small $R e$}

\subsection{Zero-order approximation}

In this paper we shall consider the solution generated under the following conditions:

$$
S l R e \ll \frac{a^{2}}{L^{2}}, \quad S l \gg R e .
$$

The first condition is equivalent to the condition $t_{v} \ll \tau$ mentioned above while the second one means that the transient inertia term prevails over the convective inertia in (15). A similar limitation has been already considered in a number of works (Leichtberg et el. 1976; Lovalenti \& Brady 1993), corresponding to an isolated particle case, as well as in the multi-particle problem (Pieńkowska 2001). Another restriction concerns the size of the assemblage, which should remain relatively compact over durations of interest, i.e. $L(t) \sim O(L(0)) \ll a(S l R e)^{-1 / 2}$. Although, there is no general criterion that would guarantee that inter-particle spacing remains bounded, there is, nevertheless, a number of well-studied cases, where a collection of particles remains compact for times far exceeding the typical viscous time. For example, the existence of the compact triplet in the 3-particle gravitational settling in a vertical plane for an extremely wide range of initial configurations was shown to be a consequence of a transient chaotic saddle in the phase space (Jánosi et al. 1997). Some studies concern periodic and quasi-periodic dynamics of 3-particle sedimentation (Hocking 1964; Caflisch et al. 1988; Golubitski, Krupa \& Lim 1991; Snook, Briggs \& Smith 1997), while Durlofsky, Brady \& Bossis (1987) constructed periodic solutions for configurations of four and eight particles that can be anticipated solely from the symmetry of Stokes equations.

The zero-order approximation is the quasi-steady solution of (15)-(23) under the assumption of $R e=0$. In this case, the dependence of forces (6) and torques (7) on the particle positions and velocities is local in time, while the dependence on the velocities is linear. Thus, when the solution of problem (i) is known, the particle velocities can be found by solving a system of linear algebraic equations. The evolution of the assemblage configuration can be obtained by solving a system of ordinary differential equations (8). This is the standard procedure to solve the mobility problem under the quasi-stationary approximation, and, in principle, it can also be employed for studying 
nonlinear cases. However, for non-zero Re the dependence of forces and torques on velocities is not linear and, moreover, it is not local, i.e. the force acting on a particle depends not only on instantaneous positions and velocities but also on the history of the process. This makes the inversion of the resistance problem a rather complicated task. In the present paper we consider the mobility problem directly without solving an auxiliary resistance problem.

Under the approximation of quasi-stationary Stokes flow, the positions of the particles $\boldsymbol{Z}_{i}$ vary with time; although the velocity field is found as a solution of a non-evolutionary problem, it depend on time parametrically via the evolution of the problem domain. Of course, the quasi-steady solution cannot satisfy the initial condition (20)-(21) and a smaller timescale of $O\left(t_{v}\right)$ exists, representing the initial period of rapid acceleration from rest. Re-scaling of the dimensionless time as $t^{*}=(S l R e)^{-1} t$ shows that in the initial period all three terms in (22) are of the same order of magnitude, the unsteady term in (15) is $O(1)$, while the nonlinear convective term is of $O(R e)$ and can be neglected. Therefore during this initial period the unsteady inertial, virtual-mass and the Basset forces would be equally operative (Leichtberg et al. 1976). The right-hand side of (19) becomes of $O(R e)$ as well hence the change in particle positions can be neglected. Thus, the initial transient period can be described by the fully linearized problem studied by Leichtberg et al. (1976) and by Lovalenti \& Brady (1993), corresponding to an isolated particle, as well as in the multi-particle problem (Pieńkowska 2001).

In what follows we shall focus on the long-time behaviour of the assemblage, $t \gg t_{v}$, when the quasi-steady velocity and pressure fields in the vicinity of the swarm have already developed. Therefore, we neglect the initial short transient motion and we do not attempt to satisfy the initial conditions exactly everywhere. More specifically, we are interested in the leading-order inertial corrections to the quasi-steady particle velocities $\boldsymbol{u}_{i}=\boldsymbol{u}_{i}^{0}+\epsilon \boldsymbol{u}_{i}^{1}+o(\epsilon), \epsilon(S l, R e) \ll 1$.

It follows from (12) that the undisturbed pressure can be readily found for an arbitrary $R e$ as

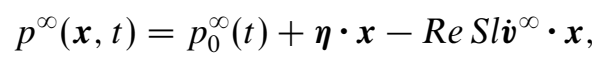

where $p_{0}^{\infty}(t)$ is an arbitrary time-dependent uniform pressure. For $R e=0$ the problem formulated in (15)-(23) is a Stokes problem. Using the boundary integral representation of the velocity field, $\boldsymbol{v}^{0}$, corresponding to $R e=0$, and eliminating the double layer integral (Pozrikidis 1992), we arrive at

$$
\boldsymbol{v}^{0}=-\frac{1}{8 \pi} \oint_{S}\left[\boldsymbol{\sigma}^{0}(\boldsymbol{y}) \cdot \boldsymbol{n}+\boldsymbol{\sigma}_{0}^{\infty}(\boldsymbol{y}) \cdot \boldsymbol{n}\right] \cdot \mathscr{G}(\boldsymbol{x}-\boldsymbol{y}) \mathrm{d} s(\boldsymbol{y}),
$$

where $\boldsymbol{\sigma}_{0}^{\infty}$ denotes a stress corresponding to the solution of a homogeneous Stokes equation with the undisturbed velocity $\boldsymbol{v}^{\infty}, S=\bigcup_{i} \Gamma_{i}$ is a multiply-connected solid surface and $\mathscr{G}$ is the Oseen-Burgers tensor or Stokeslet with components

$$
\mathscr{G}_{i j}(\boldsymbol{x})=\frac{\delta_{i j}}{r}+\frac{x_{i} x_{j}}{r^{3}} .
$$

Note that (26) is valid for an arbitrary Stokesian velocity field $\boldsymbol{v}^{\infty}$. For the special case of a spatially uniform flow, $\boldsymbol{\sigma}_{0}^{\infty} \cdot \boldsymbol{n}$ is just a constant pressure, $-p_{0}^{\infty} \boldsymbol{n}$, of no dynamic significance and does not contribute to the the integral in (26) since $\mathscr{G}$ is solenoidal. Far from the swarm, $|\boldsymbol{x}| \gg|\boldsymbol{y}|$, the leading term of the disturbance field can be found as in Kim \& Karrila (1991), by using the multi-pole expansion of the integral 
representation for the velocity and pressure fields,

$$
\begin{aligned}
\boldsymbol{v}^{0} & =-\frac{\boldsymbol{F}^{0} \cdot \mathscr{G}(\boldsymbol{x})}{8 \pi}+O\left(|\boldsymbol{x}|^{-2}\right), \\
p^{0} & =-\frac{\boldsymbol{F}^{0} \cdot \mathscr{P}(\boldsymbol{x})}{8 \pi}+O\left(|\boldsymbol{x}|^{-3}\right),
\end{aligned}
$$

where $\mathscr{P}$ is the pressure vector with components $\mathscr{P}_{i}=2 x_{i} / r^{3}$ and

$$
\boldsymbol{F}^{0}=\oint_{S} \boldsymbol{\sigma}^{0} \cdot \boldsymbol{n} \mathrm{d} s
$$

is a net Stokes drag force exerted by the fluid on the particles.

The leading term of the disturbance in (27) represents the velocity field induced by a Stokeslet of strength $\boldsymbol{F}^{0}$ at $r=0$ and it is present if particles and fluid exert a net force on each other. Using (22) and (12) it follows that at vanishing $R e$

$$
\sum_{i} \oint_{\Gamma_{\mathrm{i}}}\left(\boldsymbol{\sigma}^{0}+\boldsymbol{\sigma}^{\infty}\right) \cdot \boldsymbol{n} \mathrm{d} s=\sum_{i} \oint_{\Gamma_{\mathrm{i}}}\left(\boldsymbol{\sigma}^{0}-(\boldsymbol{\eta} \cdot \boldsymbol{x}) \boldsymbol{I}\right) \cdot \boldsymbol{n} \mathrm{d} s+O(R e)=-\boldsymbol{\eta}(t) \sum_{i} \tilde{m}_{i} .
$$

Therefore, taking into account that

$$
\oint_{\Gamma_{\mathrm{i}}}(\boldsymbol{\eta} \cdot \boldsymbol{x}) \boldsymbol{n} \mathrm{d} s=\widetilde{m}_{i} \lambda_{i}^{-1} \boldsymbol{\eta}
$$

where $\lambda_{i}$ denotes the dimensionless density of the $i$ th particle non-dimensionalized by $\rho$, we finally arrive at

$$
\boldsymbol{F}^{0}=\sum_{i} \oint_{\Gamma_{\mathrm{i}}} \boldsymbol{\sigma}^{0}(\boldsymbol{y}) \cdot \boldsymbol{n} \mathrm{d} s(\boldsymbol{y})=-\boldsymbol{\eta}(t) \sum_{i} \tilde{m}_{i}\left(1-\lambda_{i}^{-1}\right) .
$$

Note that all the identities above are valid for any Stokes flow irrespective of what boundary conditions are specified on the particle boundary. Thus, they are equally applicable to both problems (i) and (ii). For the mobility problem (ii), obviously the disturbance flow is force free if there are no body forces $(\boldsymbol{\eta}=0)$ or when the particles are neutrally buoyant $\left(\lambda_{i}=1\right)$.

Consider now the case of small but non-zero Re. Our goal is to construct the leadingorder correction to the quasi-stationary solution outlined above under limitations (24). It is obvious that a regular expansion of the velocity field in integer powers of $R e$ breaks down since $\partial \boldsymbol{v}^{0} / \partial t$ decays as $r^{-1}$ far from the assemblage and thus the first correction of the velocity field, $\boldsymbol{v}^{1}$, does not possess solutions that are bounded at infinity. Thus, a singular perturbation method will be employed to construct a uniformly valid asymptotic expansion of the solution. Following a well-established procedure (van Dyke 1975) we first construct an outer expansion of the velocity and pressure fields.

\subsection{The first term of the outer expansion}

Let $\boldsymbol{\xi}=\varepsilon \boldsymbol{x}, \boldsymbol{V}(\boldsymbol{\xi}, t)=\boldsymbol{v}\left(\varepsilon^{-1} \boldsymbol{\xi}, t\right), P(\boldsymbol{\xi}, t)=p\left(\varepsilon^{-1} \boldsymbol{\xi}, t\right)$, where $0<\varepsilon(S l, R e) \ll 1$ is some small parameter. In terms of the outer spatial variables equation (15) becomes

$$
R e\left(S l \frac{\partial \boldsymbol{V}}{\partial t}+\varepsilon \boldsymbol{V} \cdot \nabla_{\xi} \boldsymbol{V}+\varepsilon \boldsymbol{f}\left(\boldsymbol{V}, \boldsymbol{V}_{\infty}\right)\right)=-\varepsilon \nabla_{\xi} P+\varepsilon^{2} \Delta_{\xi} \boldsymbol{V}
$$


We assume that the outer velocity and pressure fields have the following asymptotic expansions:

$$
\left.\begin{array}{l}
\boldsymbol{V}=f_{0}(\varepsilon) \boldsymbol{V}^{0}+f_{1}(\varepsilon) \boldsymbol{V}^{1}+\ldots, \\
P=\varepsilon\left(f_{0}(\varepsilon) P^{0}+f_{1}(\varepsilon) P^{1}+\ldots\right),
\end{array}\right\}
$$

where $\lim _{\varepsilon \rightarrow 0} f_{n+1} / f_{n}=0$ and the functions $\boldsymbol{V}^{n}$ and $P^{n}$ vanish as $|\boldsymbol{\xi}| \rightarrow \infty$. Then, with the choice $\varepsilon=\sqrt{S l R e}$ the first term of the outer expansion should satisfy the transient Stokes equation

$$
\frac{\partial \boldsymbol{V}^{0}}{\partial t}=-\nabla_{\xi} P^{0}+\Delta_{\xi} \boldsymbol{V}^{0}
$$

$f_{0}(\varepsilon)$ can be readily obtained from the matching condition, requiring that

$$
\begin{aligned}
& \lim _{|\xi| \rightarrow 0} f_{0}(\varepsilon) \boldsymbol{V}^{0}(\varepsilon \boldsymbol{x}, t)=\lim _{|\boldsymbol{x}| \rightarrow \infty} \boldsymbol{v}^{0}=-\frac{\boldsymbol{F}^{0} \cdot \mathscr{G}(\boldsymbol{x})}{8 \pi}, \\
& \lim _{|\xi| \rightarrow 0} \varepsilon f_{0}(\varepsilon) P^{0}(\varepsilon \boldsymbol{x}, t)=\lim _{|\boldsymbol{x}| \rightarrow \infty} p^{0}=-\frac{\boldsymbol{F}^{0} \cdot \mathscr{P}(\boldsymbol{x})}{8 \pi} .
\end{aligned}
$$

Thus, it follows from the above matching conditions that $f_{0}=\varepsilon$.

Let $\widehat{V}^{0}(\xi, s)$ and $\widehat{P}^{0}(\xi, s)$ be the Laplace images of the first term of the outer velocity and pressure fields, respectively, where $s$ is a Laplace variable. It can be readily verified that the fundamental solution of the transient Stokes equation with the singularity as $\xi \rightarrow 0$ corresponding to the varying point force (Kim \& Karrila 1991), given by

$$
\widehat{\boldsymbol{V}}^{0}(\boldsymbol{\xi}, s)=-\frac{\widehat{\boldsymbol{F}}^{0} \cdot \widehat{\mathscr{G}}(\xi ; s)}{8 \pi}, \quad \widehat{\boldsymbol{P}}^{0}(\boldsymbol{\xi}, s)=-\frac{\widehat{\boldsymbol{F}}^{0} \cdot \widehat{\mathscr{P}}(\xi ; s)}{8 \pi},
$$

satisfies the above equation and the matching conditions (34)-(35). Here $\widehat{\mathscr{G}}(\xi ; s)$ denotes a transient Oseen tensor with components

$$
\begin{aligned}
\widehat{\mathscr{G}}_{i j}(\xi ; s)=\frac{4}{\varrho^{3} s^{2}}[1-(1 & \left.+\varrho \sqrt{s}) \mathrm{e}^{-\varrho \sqrt{s}}\right] \frac{\xi_{i} \xi_{j}}{\varrho^{2}} \\
& +\frac{2}{\varrho^{3} s^{2}}\left[\left(1+\varrho \sqrt{s}+\varrho^{2} s\right) \mathrm{e}^{-\varrho \sqrt{s}}-1\right]\left(\delta_{i j}-\frac{\xi_{i} \xi_{j}}{\varrho^{2}}\right),
\end{aligned}
$$

and

where $\varrho=\varepsilon r=|\xi|$.

$$
\widehat{\mathscr{P}}_{i}(\xi ; s)=\mathscr{P}_{i}(\xi)=2 \frac{\xi_{i}}{\varrho^{3}},
$$

Expanding $\varepsilon \boldsymbol{v}^{0}$ near $\varrho=0$, then re-writing it in terms of the inner spatial variables up to $O(\varepsilon)$ and transforming back to the time domain, $t$, we find the outer(inner) limit of the inner(outer) solution as

$$
\boldsymbol{V}=-\frac{1}{8 \pi}\left(\boldsymbol{F}^{0} \cdot \mathscr{G}(\boldsymbol{x})-\varepsilon \frac{4}{3 \sqrt{\pi}} \int_{-\infty}^{t} \frac{\dot{\boldsymbol{F}}^{0}(\zeta)}{(t-\zeta)^{1 / 2}} \mathrm{~d} \zeta\right)+o(\varepsilon)
$$

where we assumed that $\boldsymbol{F}^{0}(-\infty)=0$, which indicates that we neglect the influence of the short-lived initial transient on the long-time behaviour. The second term of $O(\varepsilon)$ is a generalized Basset inertial term, since it accounts for all inter-particle interactions. For instance, we will show in $\S 3.4$ that this term has a non-zero value in the case of a steady translation of particles towards each other, which is a case when the classical 
Basset contribution is zero. It can be readily shown that in the case of a single particle moving due to prescribed forcing $\boldsymbol{F}^{0}(t)$ this expression will give rise to a classical Basset integral (Kim \& Karrila 1991).

The form of (37) and (32) suggests that the second term of the inner expansion should be of the order of $\varepsilon=(S l R e)^{1 / 2}$,

$$
(\boldsymbol{v}, p)=\left(\boldsymbol{v}^{0}, p^{0}\right)+\varepsilon\left(\boldsymbol{v}^{1}, p^{1}\right)+\ldots
$$

A similar expansion is expected for the variables $\boldsymbol{U}_{i}$ and $\boldsymbol{\Omega}_{i}$.

\subsection{The second term of the inner expansion}

For the mobility problem (ii), the second term in the inner expansion of the velocity and pressure fields should satisfy the Stokes equation and the following conditions:

$$
\begin{array}{cl}
-\nabla p^{1}+\Delta \boldsymbol{v}^{1}=0, & \boldsymbol{x} \in \mathscr{D}, \\
\nabla \cdot \boldsymbol{v}^{1}=0, & \boldsymbol{x} \in \mathscr{D}, \\
\boldsymbol{v}^{1}=\boldsymbol{u}_{i}^{1}(t), & \boldsymbol{x} \in \Gamma_{i}, \\
\boldsymbol{v}^{1} \rightarrow(8 \pi)^{-1} \boldsymbol{b}(t) & \text { at } \quad|\boldsymbol{x}| \rightarrow \infty,
\end{array}
$$

where

$$
\boldsymbol{b}(t)=\frac{4}{3 \sqrt{\pi}} \int_{-\infty}^{t} \frac{\dot{\boldsymbol{F}}^{0}(\zeta)}{(t-\zeta)^{1 / 2}} \mathrm{~d} \zeta
$$

and where the boundary conditions (40) are applied at the disturbed particle surfaces, $\Gamma_{i}(t)$, corresponding to their perturbed positions, $\boldsymbol{Z}_{i}(t)=\boldsymbol{Z}_{i}^{0}(t)+\varepsilon \boldsymbol{Z}_{i}^{1}(t)$. Given that particle inertia contributes the higher-order corrections, i.e. when $\left|\widetilde{m}_{i} \dot{\boldsymbol{U}}_{i}\right| \sim\left|{\widetilde{I_{i}}}_{i} \dot{\boldsymbol{\Omega}}_{i}\right|=$ $O(1)$, the dynamic force and torque balances (22)-(23) at $O(\varepsilon)$ give

$$
\begin{aligned}
& \oint_{\Gamma_{\mathrm{i}}} \boldsymbol{\sigma}^{1} \cdot \boldsymbol{n} \mathrm{d} s=0, \\
& \oint_{\Gamma_{\mathrm{i}}} \boldsymbol{r}_{i} \times\left(\boldsymbol{\sigma}^{1} \cdot \boldsymbol{n}\right) \mathrm{d} s=0 .
\end{aligned}
$$

Thus, the mobility problem (ii) formulated in (38)-(41) has a simple solution $\boldsymbol{v}^{1}=$ $\boldsymbol{u}_{i}^{1}=(8 \pi)^{-1} \boldsymbol{b}(t), p^{1}=$ Const, which satisfies (43)-(44) on any $\Gamma_{i}(t)$. Thus, the firstorder effect of fluid inertia on the motion of freely suspended particles results in an additional translational velocity, which is the same for all particles, regardless of the instantaneous configuration and velocities. Note that in the classical case of gravitational settling of particles in a constant gravity field, given that the assemblage remains compact during its fall, $L \ll \varepsilon^{-1}$, it follows from the conclusions of $\S 3.1$ that $\boldsymbol{F}^{0}$ is a constant vector and, therefore, $\boldsymbol{b}(t)=\mathbf{0}$ in (41). Thus, the problem (38)-(43) has only a trivial solution with $\boldsymbol{u}^{1}=\mathbf{0}$ and it is anticipated that, in this particular case, the first effect of the fluid inertia is of the higher order of $O\left(\varepsilon^{2}\right)$.

When a swarm of non-neutrally buoyant particles, $\lambda_{i} \neq 1$, is submerged into a harmonically oscillating uniform background flow, $\eta(t) \sim \mathrm{e}^{\mathrm{i} \omega t}$, with low oscillation frequency, $R e=\omega a^{2} / v \ll 1$, the result will be a periodic correction to the particle velocities, $\boldsymbol{u}^{1} \sim \varepsilon(1-\mathrm{i}) \mathrm{e}^{\mathrm{i} \omega t} / \sqrt{\omega}$ in the long-time limit. Although there is a phase lag in the inertia-induced particle response, it does not contribute to the departure in particle spacings, $Z_{i}^{1}(t)$, from those predicted by the zero-Re theory at $O(\varepsilon)$. 


\subsection{Motion with prescribed velocities}

In the previous section we have shown that the temporal change in the domain geometry does not always perturb the quasi-steady zero-Re solution at $O(\varepsilon)$. An assemblage of freely suspended particles does not experience a Basset force at this order and the inertia-induced correction to their velocities is of $o(\varepsilon)$. On the other hand, for the resistance problem (i), when the particle velocities, $\boldsymbol{u}_{i}(t)$, are prescribed the aforementioned Basset force may be operative. To demonstrate this idea, consider the time-dependent motion of a swarm in a fluid which is quiescent at infinity, $\boldsymbol{v}_{\infty}, p_{\infty}=0$, in the absence of body forces, $\boldsymbol{\eta}=0$. The hydrodynamic force $\boldsymbol{F}_{i}$ exerted by the fluid on the $i$ th particle is given by

$$
\boldsymbol{F}_{i}=\oint_{\Gamma_{\mathrm{i}}} \boldsymbol{\sigma} \cdot \boldsymbol{n} \mathrm{d} s+O(\operatorname{Re} S l),
$$

where the correction corresponds to the magnitude of the particle inertia.

The zero-order approximation of this problem is the solution of (15)-(21) for a given set of values, $\boldsymbol{u}_{i}(t)$. The first term in the outer expansion has exactly the same form as in (37) while the second term of the inner expansion of the velocity and pressure fields should satisfy (38)-(41) with $\boldsymbol{u}_{i}^{1}=\mathbf{0}$, where $\boldsymbol{b}(t)$ is given by (42), while the boundary conditions are applied at the particle surfaces, $\Gamma_{i}^{0}(t)$ corresponding to their known positions, $\boldsymbol{Z}_{i}(t)=\boldsymbol{Z}_{i}^{0}(t)$. The $O(\varepsilon)$ correction to the zero-Re drag force acting on $i$ th particle is

$$
\boldsymbol{F}_{i}^{1}=\oint_{\Gamma_{i}^{0}} \boldsymbol{\sigma}^{1} \cdot \boldsymbol{n} \mathrm{d} s
$$

It is readily seen that $\boldsymbol{F}_{i}^{1}$ is identical to the Stokes drag force exerted on the $i$ th particle by the uniform time-dependent flow, that equals $(8 \pi)^{-1} \boldsymbol{b}(t)$ at infinity, past a fixed assemblage of particles.

As an example, let us calculate the generalized Basset force arising in the co-axial steady approach of two particles, $\boldsymbol{u}_{i}(t)=U_{i} \boldsymbol{e}$, where $U_{i}$ are constants. In the Stokes approximation the resistance problem corresponding to the uniform co-axial flow past two separated equal spheres was first solved exactly in bi-spherical coordinates by Stimson \& Jeffery (1926). Using bi-spherical coordinates, the axial components, $F_{i}^{0}, F_{i}^{1}$ and $b$, can be found as functions of the dimensionless separation distance, $Z(t)=Z_{1}(t)-Z_{2}(t)$ by the same technique. Since the solution at leading order is quasi-stationary, i.e. it depends on time via the evolution of the problem domain and the Basset inertial term, (42) can be written as

$$
b(t)=\frac{4}{3 \sqrt{\pi}} \int_{-\infty}^{t} \frac{\dot{F}^{0}(\tau)}{(t-\tau)^{1 / 2}} \mathrm{~d} \tau=\frac{4}{3 \sqrt{\pi}} \int_{-\infty}^{t} \frac{\mathrm{d} F^{0}(Z(\tau))}{\mathrm{d} Z} \frac{\dot{Z}}{(t-\tau)^{1 / 2}} \mathrm{~d} \tau,
$$

where $F^{0}=\sum_{i=1}^{2} F_{i}^{0}$.

In figure 1 we present the results of calculations for $b(t)$ for two spheres with a radius ratio $R=a_{2} / a_{1}=3.5$ for four different modes: $(a)$ the smaller particle approaches the fixed larger one with the velocity $U_{1}=1, Z_{0}=30 ;(b)$ the smaller particle moves away from the fixed larger particle with velocity $U_{1}=1, Z_{0}=0.1 ;(c)$ the larger particle approaches the fixed smaller one with $U_{2}=1, Z_{0}=100 ;(d)$ the larger particle moves away from the fixed smaller particle with $U_{2}=1, Z_{0}=1$. Note that in case $(a) F_{1}^{0}$ and $b(t)$ have different signs and thus fluid inertia reduces the drag force which the fluid exerts on the moving smaller particle, while the drag force on the stationary larger particle increases. In case $(b)$ the situation is opposite: $F_{1}^{0}$ 
(a)

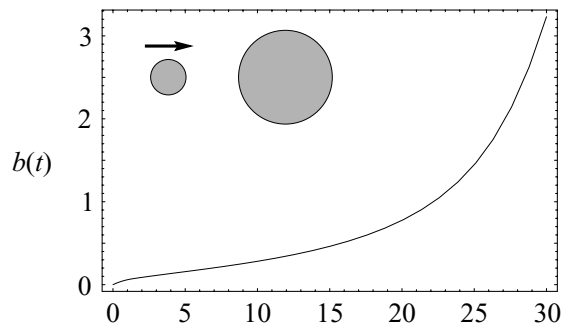

(c)

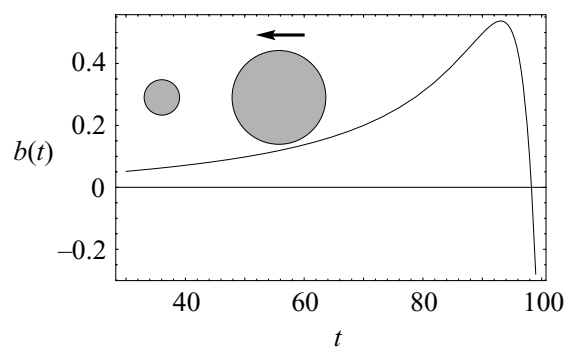

(b)

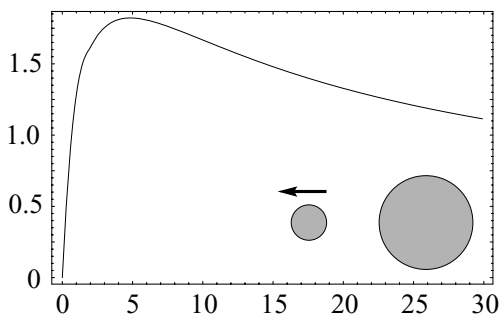

(d)

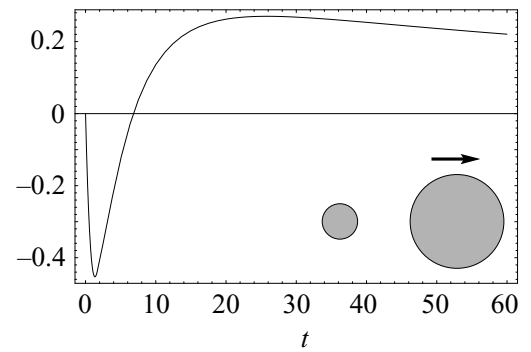

FiguRE 1. The Basset inertial term $b(t)$ in (46) vs. time for two unequal particles with a radius ratio $R=a_{2} / a_{1}=3.5$. (a) The smaller particle approaches the larger stationary one with a velocity $U_{1}=1$ and with initial separation $Z_{0}=30 ;(b)$ the small particle moves away from the stationary larger one with a velocity $U_{1}=-1$ and with initial separation $Z_{0}=0.1$; (c) the larger particle moves towards the smaller particle with a velocity $U_{2}=-1$ and the initial separation is $Z_{0}=100 ;(d)$ the larger particle moves away from the small particle with a velocity $U_{2}=1$ and where the initial separation is $Z_{0}=1$.

and $b(t)$ have the same signs and thus fluid inertia results in an increase of the drag force on the moving smaller particle, while the drag force on the stationary larger particle reduces. Although in cases $(c)$ and $(d) b(t)$ changes sign when the particles are close enough, due to non-monotonic dependence of the Stokeslet strength, $F^{0}$, on separation distance, the effect of fluid inertia on the drag force at large spacing is qualitatively the same as in $(a)$ and $(b)$, respectively. Recall that for the resistance problem for two equal particles, the four modes discussed above for the unequal particles reduce to only two modes due to symmetry: a particle moves towards or away from the neighbouring fixed particle.

Analysis of a similar example (the co-axial translation of three particles) under the limitation that the mutual positions of the particles do not change significantly with time (Pieńkowska 2001), also shows the existence of the Basset force at $O(\sqrt{S l R e})$ due to unsteadiness caused by inter-particle interactions.

\section{Discussion}

In the present paper we investigate the influence of fluid inertia on the dynamics of a finite collection of rigid spherical particles submerged in an arbitrary slowly changing uniform viscous flow in the long-time limit. Our focus is on the case of a swarm of a moderate number of particles with inter-particle separations comparable to their dimensions, when the change in mutual positions may have a substantial effect on the dynamics. For this case, the velocities of the particles in the swarm are 
comparable to the velocity of an isolated particle, which we choose for a velocity scale, $\widetilde{u}$. On the other hand our considerations are restricted to the time interval during which the swarm remains compact, i.e. the dimension of the swarm is comparable to its initial value. Since the rate of change of inter-particle separations may be either of the same order of magnitude as the individual velocities or much less, but never much greater, this time interval is $\geqslant O(\tau), \tau=a / \widetilde{u}$.

In the case $S l R e \ll a^{2} / L^{2}, S l \gg R e$, we found that the flow field possesses a tworegion structure. In the vicinity of the assemblage there exists, to a first approximation, a quasi-steady flow field region that we term the inner region and which corresponds to inertia-less motion. Far from the assemblage at $r \sim \sqrt{\nu \tau}$ there is an outer region in which the flow field is time-dependent and the transient inertia term is comparable to the viscous term. Note that this region is closer to the assemblage than the classical Oseen region in which $r \sim v / \widetilde{u}$.

The first inertia effect scales as $\sqrt{a^{2} / \nu \tau}$ and it has the form of a Basset history integral which depends on the rate of change of the net Stokes drag force exerted on the particles in the swarm by the fluid. Performing a consistent approach which takes into account the inter-particle interactions and considers the proper conditions on the instantaneous boundaries of all particles, it is found that the inertia effects associated with the unsteadiness of the flow contribute to particle velocities at $O(\sqrt{S l R e})$ as long as the disturbance velocity field possesses a time-dependent Stokeslet in the far flow field. It should be noted that this leading effect is identical for all particles in the assemblage, since it originates in the outer region. Therefore, although the effect on the particle velocities is of $O(\sqrt{S l R e})$ the leading effect on the relative spacing is restricted to only $o(\sqrt{S l R e})$. Similar solution structure and memory effects due to mutual hydrodynamic interactions were found recently by Pieńkowska (2001), for the time intervals during which no significant evolution of the mutual positions of particles takes place. The asymptotic analysis presented here is applicable to larger timescales and to systems with changing geometry. On the other hand, the theory of Pieńkowska (2001) also covers small timescales (initial transient period and high-frequency oscillations of the external forcing) that are not described by our approach.

Our analysis shows that, in case of low-frequency uniform oscillatory flow around a compact assemblage of particles, the stationary history effects are independent of the assemblage configuration and inter-particle interactions and the assemblage response is analogous to that found for a single particle of arbitrary shape (Lovalenti \& Brady 1993). However, when the total Stokes drag force exerted on particles by fluid is constant, the effect of fluid inertia on the particle velocities and spacing is of $o(\sqrt{S l R e})$. It is anticipated that the fluid inertia associated with the convective terms in the Navier-Stokes equations is likely to affect the particle velocities and spacing at $O(S l R e)$ as in the classical case of a single particle. For the important and most common case of a gravitational sedimentation of particle assemblage, the action of the Basset force is limited to an initial short-lived transient period on a viscous timescale associated with the rapid acceleration from rest. Thus, an a priori retaining of the Basset integral in the dynamic long-time force balance as in Leichtberg et al. (1976) can lead to erroneous results. Moreover, in this case, the theory developed here suggests that the first-order effect of the fluid inertia is manifested at $O(R e) \dagger$ and it

$\dagger$ When there is no imposed flow and varying body force, an appropriate choice of the typical timescale will be the Stokes timescale, $\tau=t_{S}$, which leads to $S l=1$. 
has contributions from both the outer and the inner regions. The convective Oseen terms within $\varepsilon^{2} \boldsymbol{f}\left(\boldsymbol{V}^{0}, \boldsymbol{V}^{\infty}\right)$ in (31), are of the same order of magnitude $(O(R e))$ as the unsteady terms, associated at this order with the rate of change of the stresslet within a disturbance flow. In the inner region the inertia associated with the Eulerian acceleration terms and with the convective terms would again affect the motion of the particles at $O(R e)$. These findings indicate that considering unsteady inertia terms alone, while neglecting the convective terms, as in Feng \& Joseph (1995) is not sufficient to capture correctly the deviation from the quasi-steady theory in the case of sedimenting particles. The entire fluid inertia should be taken into account in the equations of motion. The leading-order effect of fluid inertia on the settling of particles in a viscous fluid is investigated in a separate paper by Leshansky, Lavrenteva \& Nir (2003).

Another difficulty may arise when studying more general spatially inhomogeneous undisturbed flows, like linear or parabolic flows. These flow fields are particularly interesting from practical and experimental points of view. For these cases, the effect of inertia within the undisturbed flow may become important at the leading order and cannot be neglected as in the case of uniform flow. In the problem studied here the inertia of the incident flow did not enter at the leading order since $\boldsymbol{V}^{\infty}$ is bounded everywhere and thus, $\varepsilon\left|\boldsymbol{f}\left(\boldsymbol{V}^{0}, \boldsymbol{V}^{\infty}\right)\right| \ll\left|\partial \boldsymbol{V}^{0} / \partial t\right|$ in (31). The motion of a particle in a steady linear flow was studied by Saffman (1965) who found that it experiences a lift force of $O(\sqrt{R e})$ perpendicular to the flow direction. For the slowly varying linear flow, $\boldsymbol{v}^{\infty}=\mathscr{A}(t) \cdot \boldsymbol{x}$, the unsteady outer problem at the leading order should be considered (the problem formulated in this paper would be modified by adding to the left-hand side of (33) two Oseen terms: $\boldsymbol{V}^{0} \cdot \nabla_{\xi} \boldsymbol{V}^{\infty}$ and $\boldsymbol{V}^{\infty} \cdot \nabla_{\xi} \boldsymbol{V}^{0}$ ). The unsteadiness of the outer field may also result from the temporal change of the inner domain in the many-body problem even when the imposed flow is independent of time. It may lead to the modification of the inertial lift force found by Saffman (1965) for a single particle.

For a parabolic flow, $\boldsymbol{v}^{\infty}=\mathscr{B}(t): \boldsymbol{x} \boldsymbol{x}+\ldots$, it is readily seen that the Oseen terms in the outer region dominate over the unsteady inertia terms and this may lead to a completely different scaling for $\varepsilon(R e)$. For example, if the particles are neutrally buoyant, the leading term in the far disturbance field is due to a force dipole and, therefore, $f_{0}=\varepsilon^{2}$ in (32) and the Oseen terms balance the viscous terms in (31) for $\varepsilon=R e^{1 / 3}$. This is valid only in the case of an unbounded parabolic flow. For the Pouseuille flow in a pipe, the linear and quadratic terms in the outer flow are of the same order of magnitude and the expansion parameter is again $\varepsilon=\sqrt{R e_{p}}$, as was shown by Schonberg $\&$ Hinch (1989) for small values of $R e_{p}$ associated with a particle size and finite Reynolds number associated with pipe flow.

Thus, our asymptotic analysis clearly indicates that the advective terms associated with the non-uniform incident flow cannot be neglected a priori even in the case of the motion of the single particle studied by Maxey \& Riley (1983). Therefore, the expression for the force exerted on a particle in a non-uniform flow (Maxey \& Riley 1983) should be modified by adding a contribution from the undisturbed flow inertia, neglected in their analysis. The question concerning the first effect of inertia on the dynamics of an assemblage embedded in spatially non-uniform flows is yet to be answered.

This work was supported by the fund for the promotion of research at the Technion - IIT. O.M.L. acknowledges the support of the Israel Ministry for Immigrant Absorption. 


\section{REFERENCES}

Caflisch, R. E., Lim, C., Luke, J. H. C. \& Sangani, A. S. 1988 Periodic solutions for 3 sedimenting spheres. Phys. Fluids 31, 3175-3179.

Chang, E. J. \& Maxey, M. R. 1994 Unsteady flow about a sphere at low to moderate Reynolds numbers. Part 1. Oscillatory motion. J. Fluid Mech. 277, 347-379.

Coimbra, C. F. M., L'Esperance, D., Lambert, R. A., Trolinger, J. D. \& Rangel, R. H. 2004 An experimental study on stationary history effects in high-frequency Stokes flows. J. Fluid Mech. (in press).

Coimbra, C. F. M. \& Rangel, R. H. 2001 Spherical particle motion in harmonic Stokes flows. AIAA J. 39, 1673-1682.

Durlofsky, L., Brady, J. F. \& Bossis, G. 1987 Dynamic simulation of hydrodynamically interacting particles. J. Fluid Mech. 180, 21-49.

FenG, J. \& Joseph, D. D. 1995 The unsteady motion of solid bodies in creeping flows. J. Fluid Mech. 303, 83-102.

Golubitski, M., KruPA, M. \& Lim, C. 1991 Time-reversibility and particle sedimentation. SIAM J. Appl. Maths 51, 49-72.

HinCH, E. J. \& NitsChe, L. G. 1993 Nonlinear drift interactions between fluctuating colloidal particles: oscillatory and stochastic motions. J. Fluid Mech. 256, 341-401.

Hocking, L. M. 1964 The behaviour of clusters of spheres falling in a viscous fluid. J. Fluid. Mech. 20, 365-400.

Jánosi, I. M., Tél T., Wolf, D. E. \& Gallas, A. C. 1997 Chaotic particle dynamics in viscous flow: The three-particle Stokeslet problem. Phys. Rev. E 56, 2858-2868.

Kim, S. \& Karrila, S. J. 1991 Microhydrodynamics: Principles and Selected Applications. Butterworth-Heinemann.

Landau, L. D. \& Lifshitz, E. M. 1988 Hydrodynamics, vol. 6, 3rd edn. Moscow: Nauka (in Russian).

Leichtberg, S., Weinbaum, S., Pfeffer, R. \& Gluckman, M. J. 1976 A study of unsteady forces at low Reynolds number: a strong interaction theory for the coaxial settling of three or more spheres. Phil. Trans. R. Soc. Lond. 282, 585-610.

Leshansky, A. M., LaVrenteva, O. M. \& NiR, A. 2003 The weakly inertial settling of particles in a viscous fluid. Proc. R. Soc. Lond. A 459, 3079-3098.

Lovalenti, P. M. \& BRAdy, J. F. 1993 The hydrodynamic force on a rigid particle undergoing arbitrary time-dependent motion at small Reynolds number. J. Fluid Mech. 256, 561-605.

Machu, G., Meile, W., Nitsche, L. C. \& Schaflinger, U. 2001 Coalescence, torus formation and break-up of sedimenting drops: experiments and computer simulations. J. Fluid Mech. 447, 299-336.

MaXeY, M. R. \& Riley, J. J. 1983 Equation of motion for a small rigid sphere in a nonuniform flow. Phys. Fluids 26, 883-889.

MeI, R. W. \& Adrian, R. J. 1992 Flow past a sphere with an oscillation in the free-stream velocity and unsteady drag at finite Reynolds number. J. Fluid Mech. 237, 323-341.

Pieńkowska, I. T. 2001 Fluid fields due to many-body hydrodynamic interactions. Physica A 297, $13-25$.

Pozrikidis, C. 1992 Boundary Integral and Singularity Methods for Linearized Viscous Flow. Cambridge University Press.

SAfFman, P. G. 1965 The lift on a small sphere in a slow shear flow. J. Fluid Mech. 22, 385-400.

SCHONBERG, J. A. \& Hinch, E. J. 1989 Inertial migration of a sphere in Poiseuille flow. J. Fluid Mech. 203, 517-524.

Snook, I. K., Briggs, K. M. \& SMith, E. R. 1997 Hydrodynamic interactions and some new periodic structures in three particle sediments. Physica A 240, 547-559.

Stimson, M. \& Jeffery, G. B. 1926 The motion of two spheres in viscous fluid. Proc. R. Soc. Lond. A 111, 110-116.

TCHEN, C. M. 1947 Mean value and correction problems connected with the motion of small particles suspended in a furbulent fluid. Doctoral dissertation, Delft University, Hague.

van Dyke, M. 1975 Perturbation Methods in Fluid Mechanics. Parabolic Press. 